\title{
Highlighting glycosylation ways in Caryophyllaceae saponins by simplex simulation approach
}

\author{
Hammami Asma $^{a}$, Farman Muhammad $^{b}$, Semmar Nabil $^{c^{*}}$ \\ ${ }^{a}$ Institut National des Sciences Appliquées et de Technologies (INSAT), Université de Carthage, \\ Tunisia \\ ${ }^{b}$ Quaid-i-Azam University, Department of Chemistry, Islamabad 45320, Pakistan \\ ${ }^{c}$ Laboratory of BioInformatics, bioMathematics and bioStatistics (BIMS), Institut Pasteur de Tunis, \\ 1002, Université de Tunis El Manar, Tunis, Tunisia \\ *For correspondence: nabilsemmar5@gmail.com
}

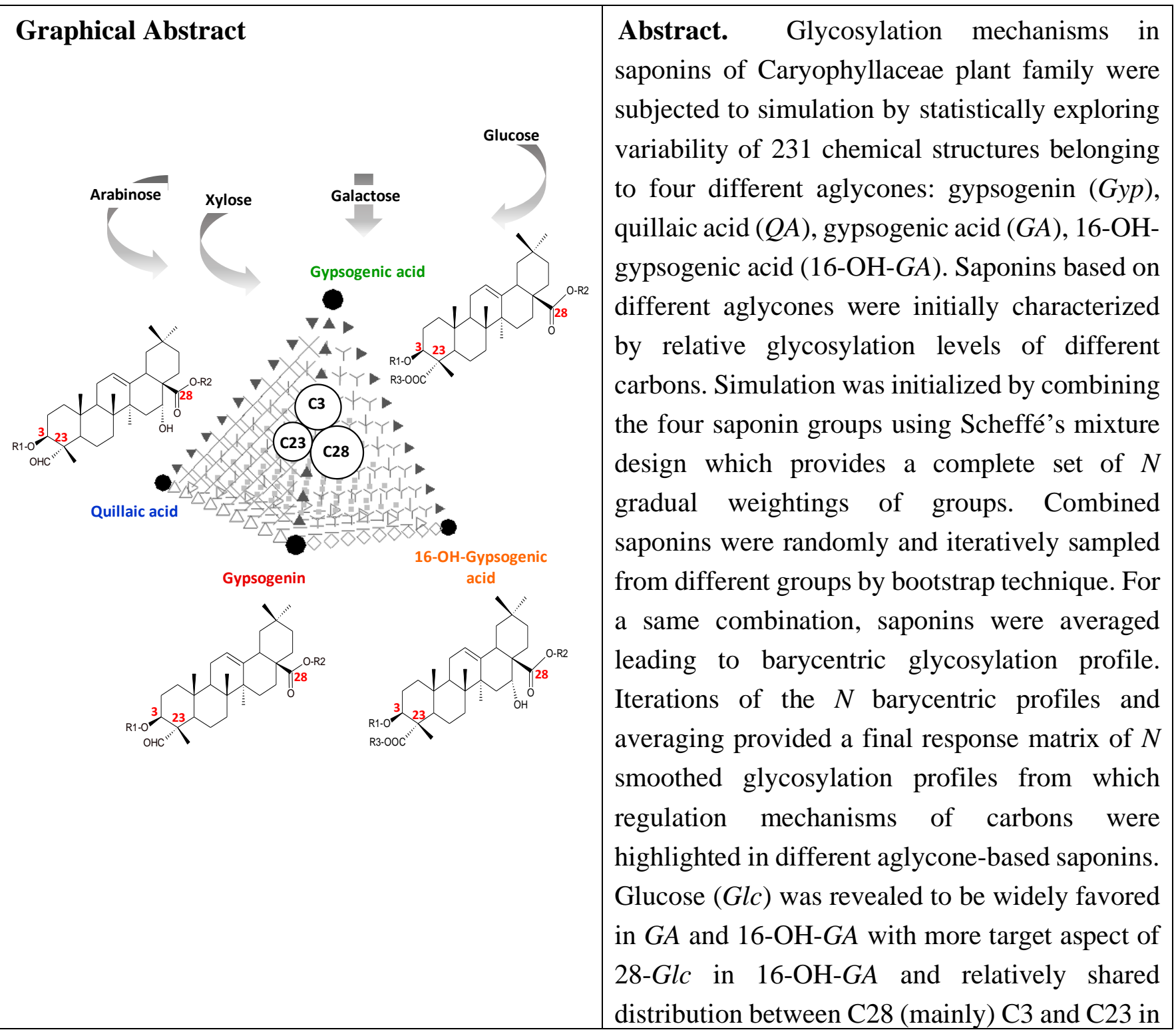


$G A$. Strong competition for galactose $(\mathrm{Gal})$ was highlighted between $\mathrm{C} 3$ and $\mathrm{C} 28$ with target aspects to 28-Gal in GA and 3-Gal in (Gyp, QA). Gyp and $Q A$ showed higher regulations of pentoses (xylose, $X y l$; arabinose, Ara) with more affinity of $G A$ for (3-Ara, 28-Xyl) and 16-OH-GA for (3-Xyl, 28-Ara). These results call for further investments in simulations of glycosylation mechanisms helping for better understanding metabolic aspects of saponins, and encouraging future analytic experiments in the field.

Introduction. Saponin synthesis is essentially based on glycosylation of sapogenin at different candidate carbons by different saccharide types with different levels. Regulation mechanisms of saponin synthesis remain limited because of the few investments in analytical experiments concerning enzymatic action modes, steric effects and regioselectivity (Tiwari et al., 2016; Moses et al., 2013; Meesapyodsuk et al., 2007; Haralampidis et al., 2002). Such mechanisms could be alternatively approached by computational way consisting in statistically exploring structural variability of saponins to highlight regulation trends between glycosylated carbons (Sarraj-Laabidi et al., 2018). For that aim, simulation simplex approach was applied to a bibliographic set of 231 Caryophyllaceae saponins to highlight aglycone- and carbon-dependent glycosylation mechanism for different saccharides.

Materials and Method. A whole set of 231 saponins was initially organized into 4 aglycone subsets: Gyp, $Q A, G A, 16-\mathrm{OH}-G A(79,80,52,20$ saponins, respectively) (Cheikh Ali et al., 2019). Saponins were characterized by their glycosylation (Gly) regulation profiles containing relative occurrence degrees of different saccharides at different carbons (Sarraj et al., 2018). Gly profiles of saponins were iteratively combined by applying Scheffé's mixture design giving a complete set of $N$ gradual weights for the $q(=4)$ groups (Scheffé, 1958). At the output of $\mathrm{N}$ iterated combinations, $N$ barycentric $G l y$ profiles were calculated resulting in smoothed data that were used for graphical analysis of regulation trends between different saccharides at different carbons under different aglycones (Fig. 1).

Results. Glc strongly characterized GA and 16-OH-GA saponins whereas Gyp and $Q A$ showed higher diversification of glycosylation at the expense of Glc. GA and 16-OH-GA were functionally differentiated by distributional and target aspects of $G l c$, respectively (Fig. 1). In GA, Glc was shared between C28 (60\%), C3 (12\%) and C23 (5\%), whereas in 16-OH-GA, Glc was mainly target to C28 (80\%). Gyp and $Q A$ were less concerned with Glc with low regulation levels (<3\%). This could indicate interactions between aglycone type and carbon position on regulation levels of Glc. Concerning Gal, GA showed relatively higher trend of 28-Gal whereas 16-OH-GA did not favor galactosylation at all the carbons. However, Gyp and $Q A$ showed target mechanism of 3-Gal at the expense of 28-Gal. Pentoses (Xyl, Ara) manifested in Gyp and $Q A$ vs negligible levels in GA and 16$\mathrm{OH}-G A$. Gyp and $Q A$ were differentiated by glycosylation positions: 28-Xyl and 3-Ara were relatively more favored in Gyp, vs 28-Ara and 3-Xyl in QA. 


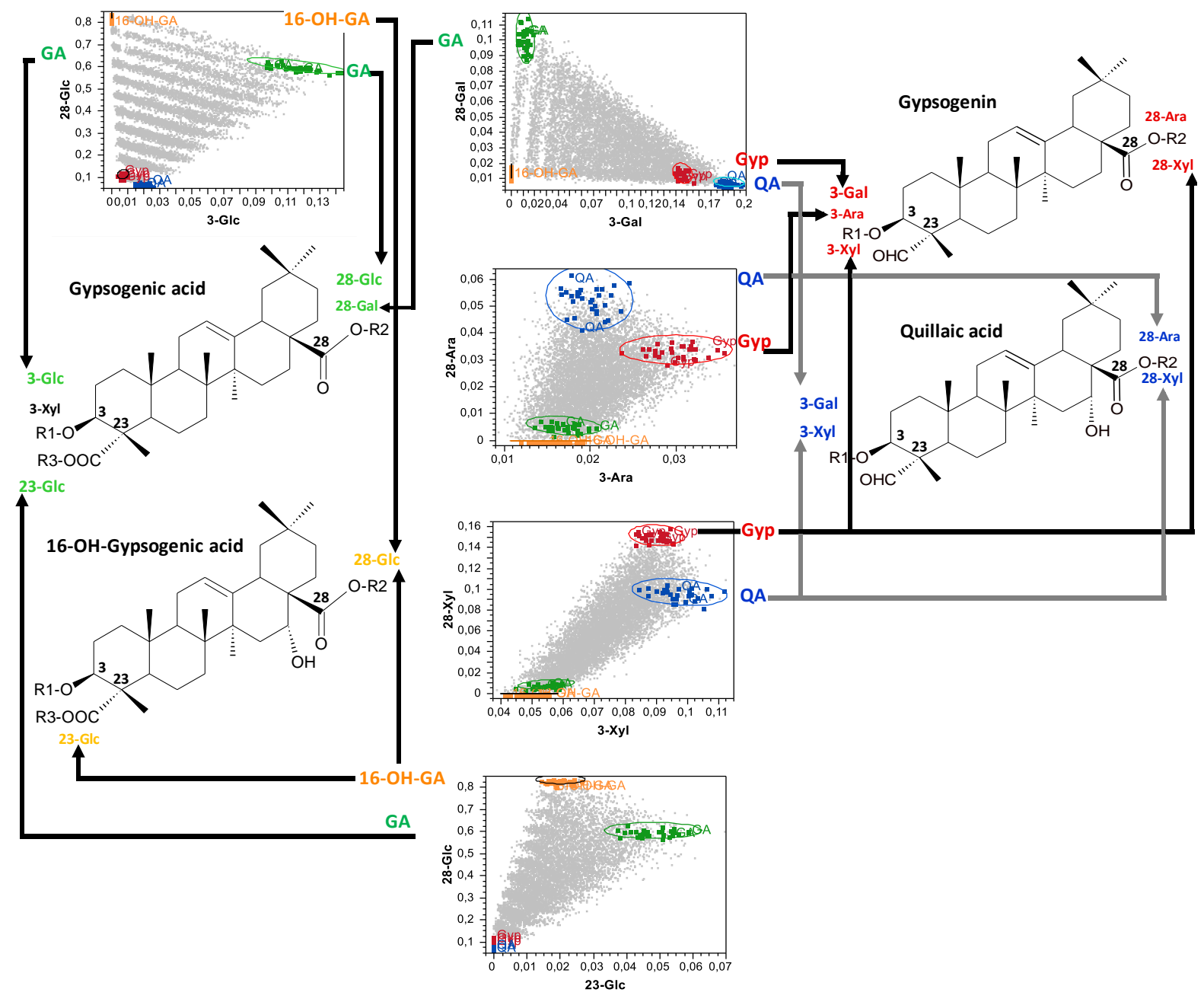

Figure 1. Smoothed plots by simplex approach highlighting regulation trends of different saccharides between different carbons in different aglycones (gypsogenin, quiallic acid, gypsogenic acid, 16-OHgypsogenic acid) of Caryophyllaceae saponins.

Conclusion. Simulation results could be indicative of differential regioselectivities concerning different saccharide types varying at both inter-molecular (between sapogenins) and intra-molecular (between carbons) scales.

\section{References}

Cheikh Ali, S., Farman, M., Lacaille-Dubois M.A., Semmar, N., 2019. Structural organization of saponins in Caryophyllaceae. Phytochemistry Reviews 18(2), 405-441.

Haralampidis, K. Trojanowska, M., Osbourn, A.E., 2002. Advances in Biotechnological Engineering \& Biotechnology, 75, 31-49.

Meesapyodsuk, D., Balsevich, J., Reed, D.W., Covello, P.S., 2007. Saponin Biosynthesis in Saponaria vaccaria. cDNAs Encoding b-Amyrin Synthase and a Triterpene Carboxylic Acid Glucosyltransferase. Plant Physiology, 143, 959-969.

Moses, T., Pollier, J., Thevelin, J.M., Goossens, A., 2013. Bioenggineering of plant (tri)terpenoids: from metabolic engineering of plants to synthetic biology in vivo and in vitro. New Phytologist 200, 27-43. 
Sarraj-Laabidi, A., Messai, H., Hammami-Semmar, A., Semmar, N., 2017. Chemometric analysis of inter- and intra-molecular diversification factors by a machine learning simplex approach. A review and research on Astragalus saponins. Current Topics in Medicinal Chemistry, 17(25), pp 2820-2848.

Scheffé, H. 1958. Experiments with mixtures. J. R. Stat. Soc. B, 20, 344-360.

Tiwari, P., Sangwana, R.S., Sangwan, N.S., 2016. Plant secondary metabolism linked glycosyltransferases: An update on expanding knowledge and scopes. Biotechnology Advances 34(5), 714-739. 